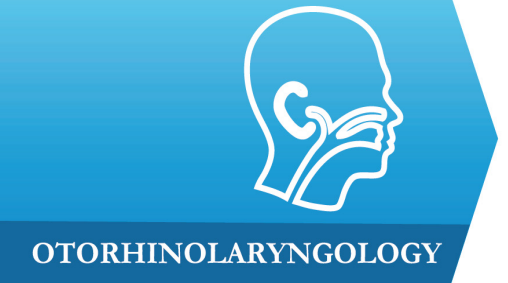

1) $2^{\text {nd }}$ Department of Otolaryngology, Iuliu Hatieganu University of Medicine and Pharmacy, ClujNapoca, Romania

2) Department of Head and Neck Surgery and Otorhinolaryngology, University Clinical Hospital of Railway Company, Iuliu Hatieganu University of Medicine and Pharmacy, Cluj-Napoca, Romania Department of Anatomy and Embryology, Iuliu Hațieganu University of Medicine and Pharmacy Cluj-Napoca

3) "Prof. C. Craciun" Electron Microscopy Laboratory, Faculty of Biology \& Geology, Babes-Bolyai University; Electron Microscopy Integrated Laboratory, National Institute for $\mathrm{R} \& \mathrm{D}$ of Isotopic and Molecular Technologies, ClujNapoca, Romania

\title{
Biofilms and inflammation in patients with chronic rhinosinusitis
}

Lavinia-Gianina Manciula ${ }^{1}$, Ionut Isaia Jeican ${ }^{2}$, Lucian Barbu Tudoran ${ }^{3}$, Silviu Albu ${ }^{1}$

\begin{abstract}
Introduction. The aim of the present study is to evaluate the presence of biofilms in patients with chronic rhinosinusitis (CRS), with or without nasal polyps, and their relationship to eosinophils and plasma cells. We compared the results with those obtained in nonCRS patients.

Methods. A total of 50 patients were included in the study, $30 \mathrm{CRSwNP}$ patients, 10 CRSsNP cases and 10 control patients who were operated for deviated septum. Biofilm detection was performed by means of H\&E staining and SEM. Eosinophil and plasma cell values were recorded and compared between groups.

Results. Biofilms were identified in 30 patients (60\%), 76.6\% (23 out of 30 ) of the CRSwNP patients, 70\% (7 out of 10) of the CRSsNP patients and none of the septoplasty patients. Eosinophil and plasma cell values were more elevated in CRS patients, being strongly correlated to biofilm presence and nasal polyposis.

Conclusion. Biofilm presence was demonstrated in many of the CRS patients, with no evidence in the control cases. Our study findings indicate that inflammatory cell counts are higher in patients with CRS compared to controls, but also more elevated in patients with polyposis. In biofilm-positive patients, eosinophil and plasma cell counts were greater than those in patients without biofilms, demonstrating the proinflammatory action of the biofilm in the sino-nasal pathology.
\end{abstract}

Keywords: chronic rhinosinusitis, biofilms, eosinophil, plasma cells, microscopy

\section{Introduction}

Chronic rhinosinusitis is one of the most common chronic medical conditions worldwide, causing significant morbidity and discomfort, leading to high financial costs. In the United States only, there is an estimate of $10 \%$ of the adult population diagnosed with CRS, with over 26 million people affected [1]. A significant percentage of the ambulatory visits result in antibiotic prescriptions, but excessive antibiotherapy recommendations can lead to adverse effects, with associated direct and indirect costs of 20 billion to 35 billion $\$$ [1]. Therefore, therapies that avoid the unnecessary use of antibiotics are sought, while making an attempt to reduce the total costs that are associated with the CRS cases.
The decreased quality of life scores of patients with chronic rhinosinusitis are often compared to those of patients with COPD or heart disease $[2,3]$, with people frequently experiencing altered mood episodes and fatigue [4]. Currently, CRS is defined as the inflammation of the nose and paranasal sinuses characterized by two or more symptoms (nasal blockage or congestion, nasal discharge, facial pain or pressure, smell reduction or loss), with a duration of more than 12 weeks and no resolution of symptoms [5], associated with highly suggestive endoscopic and CT signs.

Nowadays, chronic rhinosinusitis is considered a multifactorial disease, linked to immunological factors, local host factors, superantigens, inflammation and associated comorbidities, such as 
asthma, allergic rhinitis or Samter's triad. Based on the presence or absence of nasal polyps, two different phenotypes have been initially identified, CRS without nasal polyps (CRSsNP) and CRS with nasal polyps (CRSwNP) [6]. They have been associated with different inflammatory profiles, with CRSsNP being characterized by a neutrophilic type of inflammation, while CRSwNP was considered mainly eosinophilic. However, this description is variable among populations, as the histological study of the nasal polyps has shown that forms which are characterized by tissue eosinophilia are more frequently encountered in the western countries. Neutrophilic polyps are often described in patients from Asian countries (although a shift towards eosinophilia is possible in some areas) and in cystic fibrosis patients.

The simple classification of rhinosinusitis according to the presence or absence of nasal polyps does not describe accurately the heterogeneity of the CRS spectrum. Multiple studies have identified different clinical and biological factors which are involved in the etiology of CRS, creating the way for various disease manifestations, leading to variable treatment responses. Nowadays, CRS is considered to have multiple phenotypes and endotypes, differentiated by clinical factors, immunological factors and various biomarkers [7]. Endotyping achieves the classification of CRS cases by defining inflammatory markers, clinical features, local microbiota, nasal secretions [6] and other parameters, so that a more accurate description is obtained. Identifying these factors could lead to a more precise diagnosis, identification of potentially resistant cases and designing an individualized, endotype driven treatment. Recent research has demonstrated the existence of mixed inflammatory patterns and evidenced variations between populations [6]. Bachert et al. classified CRS, based on clinical features and relevant comorbidities into three endotypes, such as moderate type 2 inflammation, in which cases with and without polyps were included, with associated moderate asthma and recurrence risk; severe type 2, including cases with nasal polyposis (CRSwNP phenotype), with high asthma and recurrence risk and the non-type 2 inflammation, comprising cases without nasal polyps (CRSsNP phenotype), characterized by low asthma and recurrence risk [6].

The presence of mucosal biofilms in CRS, demonstrated by multiple studies, is believed to contribute to the persistence and the refractory nature of the disease. They are defined as organized communities of bacteria, enclosed in an exopolymeric matrix, composed of water, polysaccharides, nucleic acids and proteins. Biofilms are characterized by great resistance to host defence mechanisms and antibiotic therapy. Multiple studies have demonstrated unfavorable outcomes in CRS patients who developed Pseudomonas aeruginosa or Staphylococcus aureus biofilms, in terms of postoperative quality of life scores and endoscopic appearance, those cases presenting a higher risk of relapse. In CRS cases, pathogens like Haemophilus influenzae, Streptococcus pneumoniae and Moraxella catarrhalis can also form sinonasal biofilms [8]. Of all, Staphylococcus aureus is frequently associated with CRS, especially with the most severe cases and asthma patients and is associated with a type 2 inflammatory reaction. It also has the capacity to secrete superantigens which can affect the host immune response [8].

Biofilm detection can be performed by means of scanning electron microscopy (SEM), transmission electron microscopy (TEM), confocal laser scanning microscopy (CLSM) combined with live/dead staining or fluorescent in situ hybridization (FISH) [9,10], which are highly sensitive techniques. Recently, it was demonstrated that the hematoxylin and eosin staining technique can be used for biofilm identification with good accuracy and lower costs.

The objective of the present study is to detect the presence of biofilms in tissues collected from CRS and control patients and to evaluate the eosinophil and plasma cell values in the three study groups. We also aimed to determine the relationship between eosinophils and plasma cells and biofilms in the CRSwNP and CRSsNP cases.

\section{Methods}

A prospective study was designed with the purpose of determining the presence or absence of mucosal biofilm on tissue samples collected during surgery performed on CRSwNP and CRSsNP patients, as well as septoplasty patients, who served as controls. The relationship between biofilms and eosinophils, as well as plasma cells in the analyzed specimens, was also assessed. The study was approved by the local ethics committee and was performed over a period of 12 months. All patients were 18 years of age or older. The surgical interventions were conducted in a tertiary medical center.

A total of 50 patients were included in the study. The groups consisted of 30 CRSwNP patients, 10 CRSsNP patients and 10 patients with septal deviation. Exclusion criteria were sinonasal malignancy, immunodeficiency related affections, posttraumatic sinonasal injury, pregnancy, sarcoidosis or granulomatosis. Also, prior to surgery, information regarding associated asthma, allergic rhinitis or ASA intolerance was recorded. CRS patients were treated with intranasal steroids and saline lavage one month prior to the operation. Mucosa samples originated from the maxillary and/or ethmoid sinuses in the CRS groups and from the ethmoid bulla in the septoplasty groups. 
The tissue specimens were harvested intraoperatively, then fixed and prepared for scanning electron microscopy examination (SEM) and haematoxylin and eosin staining. The eosinophil and plasma cell counts were evaluated on the H\&E histologic specimens. Their numbers were assessed per high power field (HPF) and a value of 20 eosinophils/HPF was considered to be a reference value for tissue eosinophilia. The investigator who analyzed the microscopic slides was blinded to the study groups.

\section{Scanning Electron Microscopy}

Samples were fixed in glutaraldehyde $2.7 \%$ for 2 hours, washed with PBS and then with distilled water, then left to dry. Dried samples were glued to a support with silver paste and the material was further sputter coated with $10 \mathrm{~nm}$ gold. Prepared samples were analyzed using a Hitachi SU8230 scanning electron microscope at 30 $\mathrm{kV}$. All samples were compared to a control (unaffected) tissue.

\section{Hematoxylin and eosin staining}

Part of the nasal mucosa specimens were fixed in $10 \%$ formaldehyde and embedded in $15 \%$ purified gelatin for $24 \mathrm{~h}$, at $56^{\circ} \mathrm{C}$, then fixed again in $4 \%$ paraformaldehyde for $24 \mathrm{~h}$, at $20^{\circ} \mathrm{C}$. Tissue blocks were cryoprotected in a $20 \%$ saccharose solution and sectioned into $10-\mu \mathrm{m}$ slides at $-25^{\circ} \mathrm{C}$. Slides were stored at $4^{\circ} \mathrm{C}$ in $0.1 \mathrm{M}$ PBS containing $0.03 \%$ sodium azide. Two consecutive $5-\mu \mathrm{m}$ frozen cut sections were examined after conventional staining with HE.

\section{Statistical analysis}

In order to evaluate the results we used the nonparametric Kruskall-Wallis test and the Mann-Whitney U test. The statistical analysis was performed using SPSS software (SPSS Inc., Chicago, IL). A $p$ value of $<0.05$ was considered significant.

\section{Results}

The first step of the analysis was the descriptive evaluation of the variables. Frequencies and percents were computed for nominal data, along with bar charts. For the scale variables (eosinophils and plasma cells), the descriptive statistics were computed, along with the evaluation of normality. As expected, non-normal distributions were found. Consequently, comparisons were made using nonparametric testing procedures. When the groups were compared, the Mann-Whitney test was applied for two independent samples, while the KruskallWallis for more than two. Such nonparametric procedures are also applied in the case of small samples, as the present analysis.

The sample consisted of 50 patients, of which 30 (60\%) were CRSwNP patients, 10 (20\%) with CRSsNP and $10(20 \%)$ were control patients (septoplasty patients). Out of them, the biofilms were identified in 30 patients
$(60 \%$ ), in $76.6 \%$ (23 out of 30 ) of the CRSwNP patients, $70 \%$ ( 7 out of 10 ) of the CRSsNP patients and none of the septoplasty patients respectively. The distribution of the sample based on biofilm and group is presented in Figure 1. None of the control patients presented biofilms, while most of the CRSwNP and CRSsNP patients did.

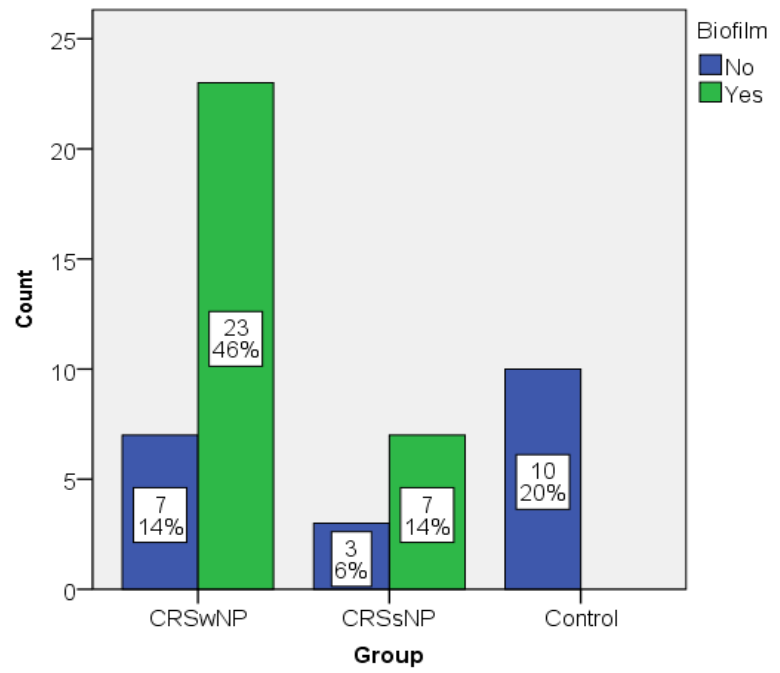

Figure 1. Distribution of the sample based on biofilm vs. Group.

Important differences were demonstrated by the descriptive statistics in the two groups, with much higher values for biofilm positive patients. For example, while the median for the eosinophils in patients without biofilm was 20 , the one for the biofilm positive patients was 80 , 4 times higher (Figure 2). Almost the same difference between the two groups was also found regarding plasma cells (15 vs 50$)$ (Figure 3 ).

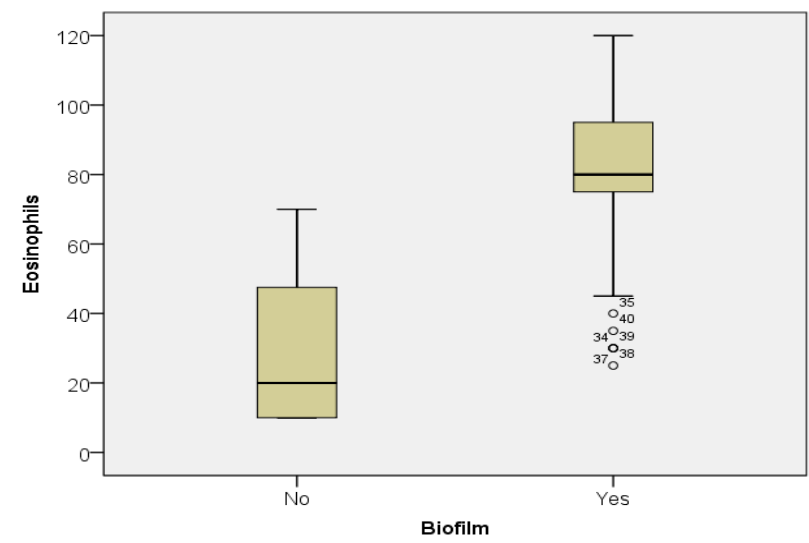

Figure 2. Eosinophil value distribution in the biofilm positive and biofilm negative patients. 


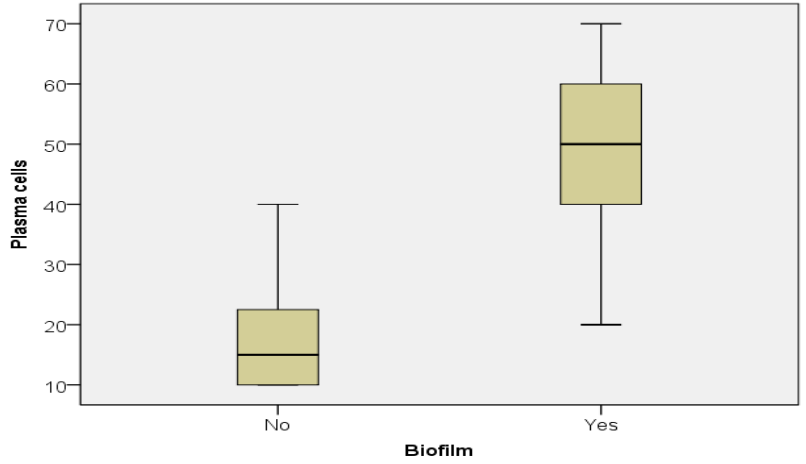

Figure 3. Plasma cell value distribution in the biofilm positive and biofilm negative patients.

In case of both variables, the values of the descriptive statistics decrease from CRSwNP towards the control group, as emphasized by the boxplots. The CRSwNP patients registered much higher values than the CRSsNP ones and much higher than controls (Figure 4 and 5).

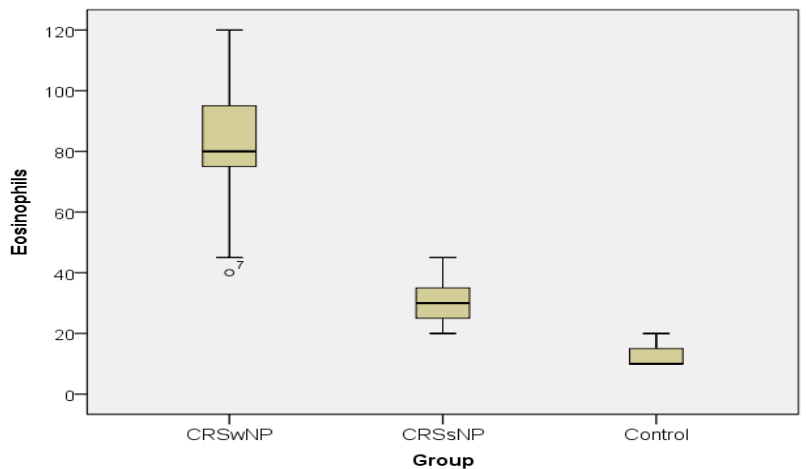

Figure 4. Eosinophil value distribution in the study groups.

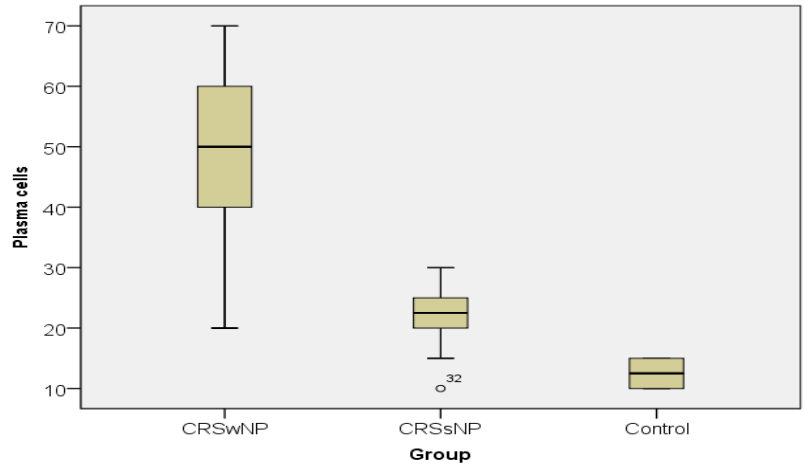

Figure 5. Plasma cell value distribution in the study groups.

The graphic representations of the mean values of eosinophils and plasma cells also illustrate the differences between biofilm positive and biofilm negative patients, but also between groups (Figure 6 and 7). Biofilm positive patients showed higher values compared to their counterparts even in the same group.

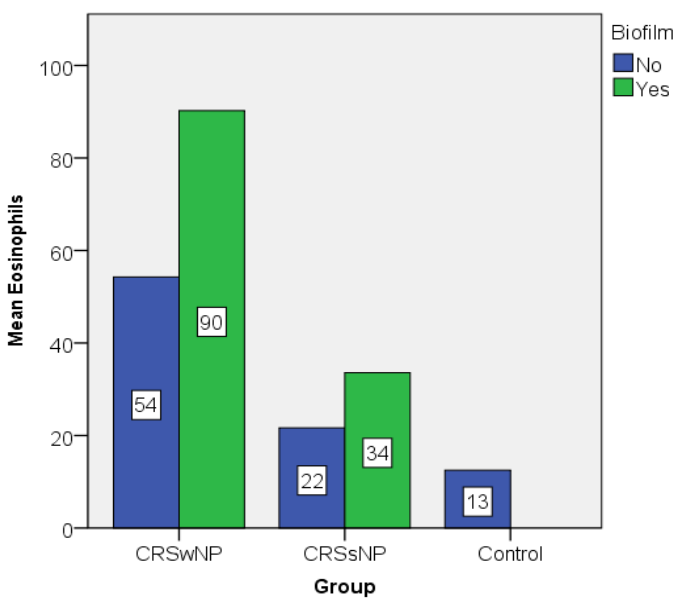

Figure 6. Eosinophil value distribution expressed as mean.

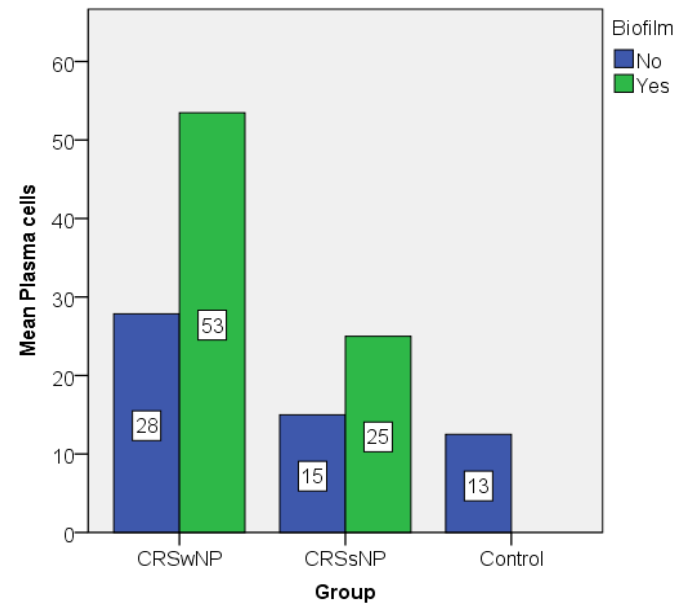

Figure 7. Plasma cell value distribution expressed as mean.

The Mann-Whitney test confirms the differences between the descriptive statistics. There are significant differences in the values and distributions of eosinophils and plasma cells between patients with and those without biofilm (Table I).

Table I. The Mann-Whitney U test results.

\begin{tabular}{l|c|c|}
\multicolumn{3}{c}{ Test Statistics ${ }^{\text {a }}$} \\
\hline & Eosinophils & Plasmocytes \\
Mann-Whitney U & 47.500 & 32.000 \\
Wilcoxon W & 257.500 & 242.000 \\
Z & -5.016 & -5.335 \\
Asymp. Sig. (2-tailed) & .000 & .000 \\
Exact Sig. (2-tailed) & .000 & .000 \\
Exact Sig. (1-tailed) & .000 & .000 \\
Point Probability & .000 & .000
\end{tabular}

a. Grouping Variable: Biofilm 
The Kruskall-Wallis test also demonstrates statistically significant differences between groups, as shown in table II.

Table II. The Kruskall-Wallis Test results.

\begin{tabular}{l|c|c|}
\multicolumn{3}{c}{ Test Statistics ${ }^{\text {a,b }}$} \\
\hline & Eosinophils & Plasmocytes \\
\hline Chi-Square & 37.419 & 33.270 \\
df & 2 & 2 \\
Asymp. Sig. & .000 & .000
\end{tabular}

a. Kruskal Wallis Test; b. Grouping Variable: Group

The Mann-Whitney test applied on the CRSwNP and CRSsNP groups evidenced statistically significant differences between the eosinophil and plasma cells values (Table III).

Table III. The Mann-Whitney U test results in the CRS groups.

\begin{tabular}{|l|c|c|}
\hline \multicolumn{3}{c|}{ Test Statistics $^{\mathrm{a}}$} \\
\hline & Eosinophils $^{\text {Plasmocytes }}$ \\
Mann-Whitney U & 2,000 & 21.000 \\
Wilcoxon W & 57.000 & 76.000 \\
Z & -4.643 & -4.057 \\
Asymp. Sig. (2-tailed) & .000 & .000 \\
Exact Sig. [2*(1-tailed Sig.)] & $.000^{\mathrm{b}}$ & $.000^{\mathrm{b}}$ \\
Exact Sig. (2-tailed) & .000 & .000 \\
Exact Sig. (1-tailed) & .000 & .000 \\
Point Probability & .000 & .000
\end{tabular}

a. Grouping Variable: Group; b. Not corrected for ties.

When comparing CRSwNP patients to controls, the differences obtained are also significant (Table IV).

Table IV. The Mann-Whitney U test results for CRSwNP and control groups analysis.

\begin{tabular}{lcc|c|}
\multicolumn{3}{c|}{ Test Statistics $^{\mathrm{a}}$} \\
\hline & Eosinophils & Plasmocytes \\
Mann-Whitney U & .000 & .000 \\
Wilcoxon W & 55.000 & 55.000 \\
Z & $-4,712$ & -4.717 \\
Asymp. Sig. (2-tailed) & .000 & .000 \\
Exact Sig. [2*(1-tailed Sig.)] & $.000^{\mathrm{b}}$ & $.000^{\mathrm{b}}$ \\
Exact Sig. (2-tailed) & .000 & .000 \\
Exact Sig. (1-tailed) & .000 & .000 \\
Point Probability & .000 & .000
\end{tabular}

a. Grouping Variable: Group; Not corrected for ties.

Similarily, significant differences in the eosinophil and plasma cell values were obtained when comparing
CRSsNP patients to controls (Table V).

Table V. The Mann-Whitney U test results for CRSsNP and control groups analysis.

\begin{tabular}{|l|c|c|}
\hline \multicolumn{3}{c}{ Test Statistics $^{\mathrm{a}}$} \\
\hline Mann-Whitney U & Eosinophils $^{\text {Plasmocytes }}$ \\
\hline Wilcoxon W & 1.000 & 10.000 \\
Z & 56.000 & 65.000 \\
Asymp. Sig. (2-tailed) & -3.773 & -3.118 \\
Exact Sig. [2*(1-tailed Sig.)] & .000 & .002 \\
Exact Sig. (2-tailed) & $.000^{\mathrm{b}}$ & $.002^{\mathrm{b}}$ \\
Exact Sig. (1-tailed) & .000 & .001 \\
Point Probability & .000 & .001 \\
\end{tabular}

a. Grouping Variable: Group; b. Not corrected for ties.

The statistical evaluation also showed that there were no significant differences regarding plasma cell values between the CRSsNP and control groups, in biofilm negative patients (Table VI).

Table VI. The Mann-Whitney U test results for CRSsNP and control groups analysis, in patients without biofilm.

\begin{tabular}{lccc}
\hline \multicolumn{3}{c}{ Test Statistics $^{\mathrm{a}}$} \\
\hline & Eosinophils & Plasmocytes \\
Mann-Whitney U & 1.000 & 10.000 \\
Wilcoxon W & 56.000 & 65.000 \\
Z & -2.520 & -.940 \\
Asymp. Sig. (2-tailed) & .012 & .347 \\
Exact Sig. [2*(1-tailed Sig.)] & $.014^{\mathrm{b}}$ & $.469^{\mathrm{b}}$ \\
Exact Sig. (2-tailed) & .010 & .318 \\
Exact Sig. (1-tailed) & .010 & .248 \\
Point Probability & .010 & .126
\end{tabular}

a. Grouping Variable: Group; b. Not corrected for ties.

The present study used the hematoxylin and eosin staining technique together with SEM in order to accurately visualize biofilms on tissue specimens collected from the sinuses at the time of surgery. On SEM examination slides, bacteria embedded in an extracellular matrix were evidenced (Figure 8). Samples examined by haematoxylin and eosin staining demonstrated the presence of small bacteria enclosed in an exopolymeric substance residing on the surface epithelium (Figure 9). The investigator noticed epithelial damage and metaplasia, cilia loss, but areas of healthy epithelium were also present. Regarding the bacterial content, single and mixed bacterial biofilms were described. As opposed to the first two groups, the control group demonstrated no biofilm presence on the examined samples. There was a good correlation between the results obtained by HE staining and the SEM examination. 

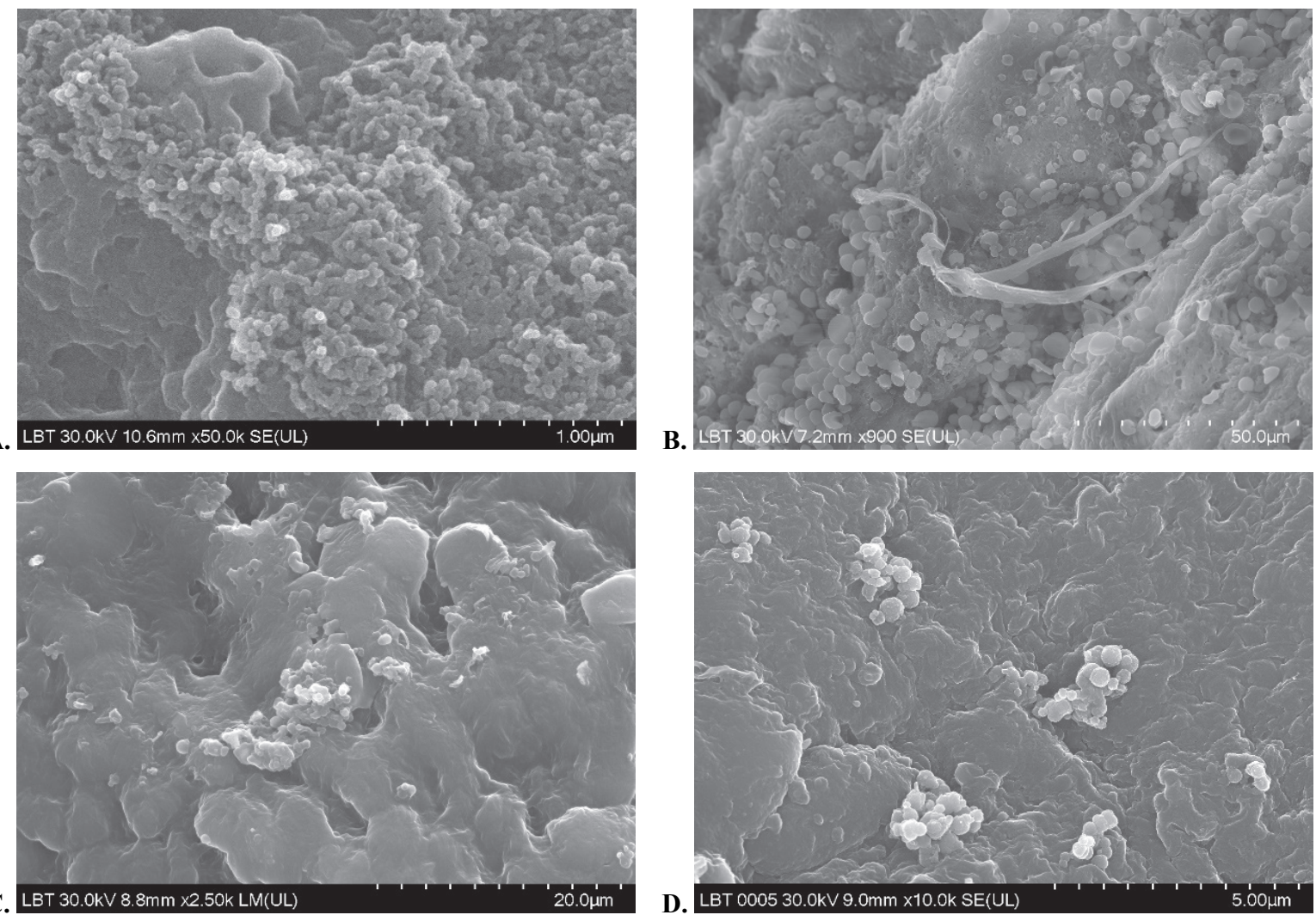

Figure 8 (A, B, C, D). Microscopy images of biofilm using SEM technique.

A.
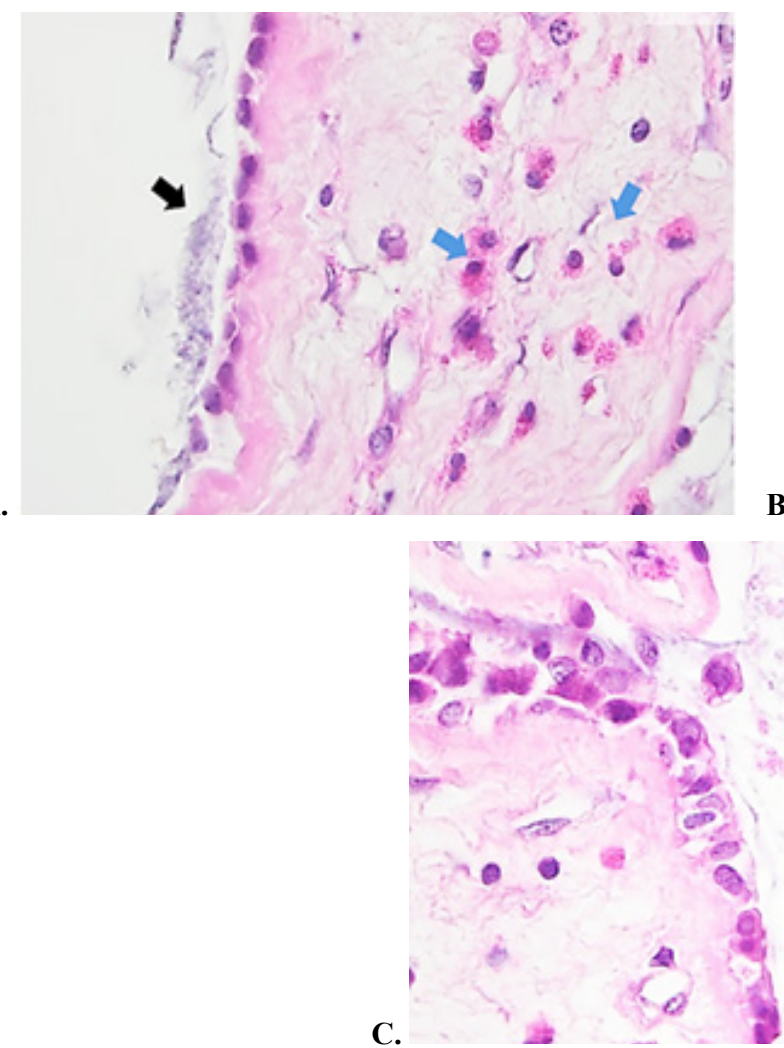

B.
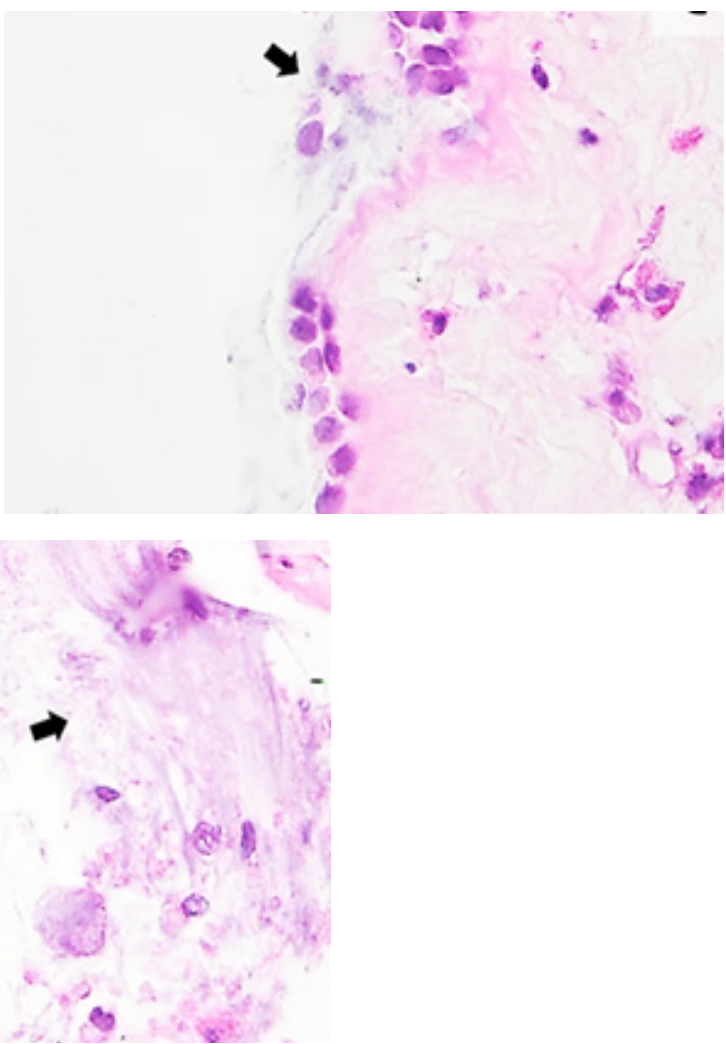

Figure 9 (A, B, C). Microscopic images of tissue samples in hematoxylin eosin staining: the biofilm presence is clearly evidenced and marked by the black arrow; eosinophils are indicated by the blue arrow. 


\section{Discussion}

Our study findings are in accordance with previous results, as eosinophil and plasma cell values were significantly higher in biofilm-positive versus biofilmnegative patients and much higher in cases with nasal polyps. The differences between the eosinophil and plasma cell counts in the CRSwNP and CRSsNP groups also reached statistical significance. For biofilm-negative patients, the differences between the inflammatory cell counts were also important amid groups, with greater values being encountered in cases with nasal polyposis. We found no significant differences in plasma cell numbers in the CRSsNP patients when compared to controls. These findings demonstrate the inflammatory burden of the CRS patients, which is greater in biofilm positive cases and much higher in biofilm positive CRSwNP groups. Tissue eosinophilia was more important in patients with nasal polyps, indicating an association with the type 2 inflammatory pathways. Previous studies also suggested that there might be an independent association between biofilms and higher levels of inflammatory cells [12].

In medicine, bacterial aggregates were described in 1977 in the lungs of cystic fibrosis patients, but it wasn't until 1981, when Costerton used the term biofilm to define bacteria embedded in a glycocalyx, that this phenomenon was named [13]. Initially, it was believed that bacteria are free floating cells, a state currently defined as planktonic. Today, planktonic bacteria are thought to be involved in the acute phases of disease. It seems that about $99 \%$ of bacteria are found in the biofilm state. Biofilms have been described in chronic conditions, such as otitis media with effusion, cholesteatoma, adenoiditis, chronic tonsillitis or cystic fibrosis [14].

The biofilm formation begins when bacteria, initially found in a planktonic state, start to develop microcolonies after adhering to an inert or biotic surface [2]. After a critical bacterial density is reached, a cell-to-cell communication phenomenon is triggered, called "quorum sensing", enabling the regulation of diverse physiological processes [2]. At one stage in their development, biofilms allow for planktonic bacteria to be released, thus colonizing new areas, causing acute infections in the patient [2]. The nature of biofilms allows pathogens to resist host defense mechanisms and antibiotic therapy. Drug resistance can be achieved in several ways, like resistance gene transmission, neutralization of the negatively charged molecules of the matrix or the dormant state of bacteria in the biofilm, which are not targeted by antibiotics [2].

Biofilm infections may activate the host and innate immune responses, which may accentuate epithelial damage [15]. They manage to mitigate the immune system's efficacy in the affected areas through different mechanisms. An agent of the innate immunity pathways, the nitric oxide (NO) has proved effective against the sinonasal microorganisms by inhibiting pathogen growth and stimulating the mucociliary clearance [16]. NO is a gas that is secreted, among others, by the upper respiratory tract and acts as a signaling molecule with potent vasodilating and antimicrobial properties. It is secreted in larger amounts by the healthy nasal mucosa and can be detected in the exhaled air [16]. In CRS patients, the levels of NO is lower, but its values are significantly decreased in primary ciliary dyskinesia and cystic fibrosis patients [16]. A recent study highlighted its antimicrobial and antibiofilm efficacy in vitro, demonstrating a synergistic action when combined with Manuka honey [1].

Bacterial biofilms have been linked to the development and persistence of chronic rhinosinusitis. Microorganisms such as Pseudomonas aeruginosa, Staphylococcus aureus, Streptococcus pneumoniae, Staphylococcus epidermidis, as well as fungus like Candida albicans and Aspergillus niger, have been isolated in biofilms [9,17-20]. Recent studies indicate that biofilms may facilitate the invasion of cells by pathogens [21]. It is noteworthy that microorganisms embedded in biofilm structures are difficult to culture by conventional methods, hence more sophisticated techniques need to be applied in order to identify these pathogens. For this reason appropriate antibiotic therapy directly targeting these bacteria is difficult to be initiated. It was demonstrated that the presence of biofilms formed by Staphylococcus aureus or Pseudomonas aeruginosa are associated with postoperative unfavorable prognosis and evolution in patients with CRS [14,22-24]. These cases were associated with worse quality of life scores and CT scan and endoscopic findings.

The development of sophisticated microscopy methods, like SEM, TEM and CSLM, allowed for biofilm structures to be evidenced and for composing microorganisms to be identified. This is especially the case with the CSLM combined with FISH technique, although there is a detection limit for only three to four species [2]. The method reproduces the three-dimensional structure of the biofilm and identifies the microbial species enclosed in the matrix [2], being a highly sensitive method of detection. In their study, Hochstim et al. [25] have demonstrated a good correlation between biofilm detection through $\mathrm{HE}$ staining and FISH analysis, making the hematoxylin and eosin staining method a cheap and reliable detection technique [9].

In the armamentarium of detection tools, in vitro techniques of evaluating the capacity of bacteria to form biofilm have been described, such as the modified Calgary Biofilm assay. The method is easily applicable and inexpensive, but might not entirely reflect the in vivo behavior of the biofilms [22]. In vitro studies have not only demonstrated the capacity of bacteria to form biofilms, but also the fact that some strains can be a lot more resistant to antibiotic therapy than those found in a planktonic state [22].

It is acknowledged now that eosinophilic inflammation has an unfavorable impact on the evolution 
of CRS cases. Previously published studies evidenced correlations between high eosinophil counts with worse CT, endoscopic and smell identification test scores $[26,27]$. Also, tissue eosinophilia has proved to be a highly reliable marker for disease recurrence [7]. Recent studies highlighted the fact that a cut off value of 55 eosinophils/ hpf is a good predictor of postoperative relapse [28]. It has been demonstrated that CRSwNP is associated with the activation of the Type 2 immune system, characterized by eosinophilic inflammation and higher IgE and IL5 in Caucasian subjects [29]. Other studies demonstrated polyp formation in CRS to be associated with increased plasma cell and eosinophil recruitment $[11,12,30]$.

In the study published by Arjomandi et al. [12], biofilms were identified in $75 \%$ of CRS cases and none in the control group. In our study, $75 \%$ of CRS cases presented biofilms, while no biofilm formation was detected in the control group. The aforementioned study also demonstrated a good correlation between biofilm presence and greater bacterial burden in biofilm positive cases [12]. The authors highlighted the higher expression of CD27 and EMBP markers, suggestive for greater CRS and plasma cell expression in biofilm positive cases, with higher values in CRSwNP compared to patients without polyps [12].

A study published by Soler et al. tried to determine whether histological measures correlate with clinical factors in patients with CRS [26]. The disease severity was evaluated by means of CT scan, endoscopy, Smell Identification Test (SIT), the Chronic Sinusitis Survey, Rhinosinusitis disability index and SF-36 General Health Survey. The histological evaluation assessed the presence of inflammatory cells, epithelial and stromal markers. They identified a positive correlation between the total eosinophil count and nasal polyposis, asthma and ASA intolerance [26]. The authors concluded that eosinophilia was associated with higher disease severity even after controlling for associated comorbidities [26].

Other studies demonstrated that biofilm positive patients had higher Lund-McKay scores and postoperative symptoms, as well as endoscopic evidence of mucosal inflammation at 8 months after FESS $[2,14]$. The findings of a study published by Psaltis et al suggested that biofilm presence could correlate with previous surgery, as its incidence was lower for patients undergoing their first procedure [14]. They also concluded that biofilms alone are not the cause for CRS, for its pathogenesis is multifactorial, but they could have a great importance in determining its recalcitrant nature [14].

The quest for alternative therapies has led investigators to elaborate different strategies in CRS cases. Topical treatments have been especially looked into, as they have lower financial costs and present a good safety profile. Methods for destabilizing the biofilm structure have been sought, thus decreasing its resistance to different therapies and immunity factors. Topical saline washes have proved their efficacy since emerging as adjuvants to topical therapies. Other substances, such as surfactants (baby shampoo) or xylitol have been used, with variable results. Baby shampoo irrigations initially showed promising results, but later studies demonstrated no difference in symptom scores in patients using hypertonic saline lavage versus $1 \%$ baby shampoo, with the latter causing bothersome nasal irritations [31,32]. Xylitol, a natural sweetener, demonstrated antibacterial and antibiofilm properties with symptomatic improvements in CRS patients [33-36]. Manuka honey is a complex compound, which achieves its antibiofilm action through multiple mechanisms, like high osmolarity, acid action, hydrogen peroxide activity and reactive compounds such as methylglyoxal (MGO) [31,37-39]. It has proved effective against bacteria, such as Staphylococcus Aureus and Pseudomonas Aeruginosa $[31,40]$. Recently, researchers have evaluated the effect of a Bioengineered Honey on the Staphylococcus aureus biofilm, observing a reduction in biofilm viability after 24 hour treatment [15]. In their study, Lim et al. have assessed the efficacy of ciprofloxacin-azithromycin sinus stent (CASS) in vitro, demonstrating a reduction in the formation of Pseudomonas Aeruginosa PAO-1 biofilms [41]. New therapeutic options like the use of topical probiotics have demonstrated good efficacy and safety in otitis media and recurrent tonsillitis [31,42].

In current clinical practice, there is a significant number of CRS cases which are resistant to optimal medical treatment and to the subsequent surgical therapies. Recognizing those cases would be of utmost importance in order to reduce hospital visits, associated medical therapy costs and multiple surgical interventions. The method of biofilm identification and histological assessment used in our study is a cheap and available technique. Therefore it could be more frequently applied in order to identify the potentially resistant CRS cases.

The present study has several limits, which will hopefully be overcome by future research. A larger number of patients selected over a longer period of time could provide more information on disease particularities and the means to associate the clinical manifestations with the histopathological aspects of the CRS cases. A correlation between the plasma cell and eosinophil counts and disease severity would help predict the evolution of the disease and identify those patients with a higher risk of postoperative recurrence. It would also guide us towards a more accurate phenotyping of CRS. A microscopic identification of the microbial species involved in the sino-nasal inflammation would be important in order to accurately describe the cases, allowing for the appropriate treatment options to be elected. Establishing the effect of the microbial metabolites on the local host defense mechanisms would also provide a clearer picture of the nature of CRS and aid in finding the appropriate methods to reduce these effects. A more 
accurate identification of the CRS biomarkers is needed in order to anticipate the disease progression and elaborate an individualized and hopefully more efficient treatment for the resistant cases.

\section{Conclusions}

Our study demonstrates the presence of biofilms in chronic rhinosinusitis and the associated inflammatory burden. It seems that CRSwNP patients are more prone to biofilm formation and also present higher eosinophil and plasma cell counts, correlating with greater disease severity. Histological demonstration of biofilm presence by means of hematoxylin and eosin staining shows promising results and correlates well with the SEM findings, becoming a cheaper and easier method for biofilm detection. Therefore it could be more easily available and frequently used in current practice.

\section{References}

1. Yang C, Mavelli GV, Nacharaju P, Li K, Cleare LG, Nosanchuk JD, et al. Novel nitric oxide-generating platform using manuka honey as an anti-biofilm strategy in chronic rhinosinusitis. Int Forum Allergy Rhinol. 2020;10:223-232.

2. Cohen M, Kofonow J, Nayak JV, Palmer JN, Chiu AG, Leid JG, et al. Biofilms in chronic rhinosinusitis: a review. Am J Rhinol Allergy. 2009;23:255-260

3. Khalid AN, Quraishi SA, Kennedy DW. Long-term quality of life measures after functional endoscopic sinus surgery. Am J Rhinol. 2004;18:131-136.

4. Martyn L, Sethia R, Chon R, Novotny L, Goodman SD, Elmaraghy $\mathrm{C}$, et al. Antibodies against the DNABII protein integration host factor (IHF) inhibit sinus implant biofilms. Laryngoscope. 2020;130:1364-1371.

5. Fokkens W, Lund V, Bachert C, Clement P, Hellings P, Holmstrom M, et al. EAACI position paper on rhinosinusitis and nasal polyps executive summary. Allergy. 2005;60:583601.

6. Ahern S, Cervin A. Inflammation and Endotyping in Chronic Rhinosinusitis - A Paradigm Shift. Medicina (Kaunas). 2019;55:95.

7. Lou H, Wang C, Zhang L. Endotype-driven precision medicine in chronic rhinosinusitis. Expert Rev Clin Immunol. 2019;15:1171-1183.

8. Fokkens WJ, Lund VJ, Hopkins C, Hellings PW, Kern R, Reitsma S, et al. European Position Paper on Rhinosinusitis and Nasal Polyps 2020. Rhinology. 2020;58(Suppl S29);1464.

9. Tóth L, Csomor P, Sziklai I, Karosi T. Biofilm detection in chronic rhinosinusitis by combined application of hematoxylin-eosin and gram staining. Eur Arch Otorhinolaryngol. 2011;268:1455-1462.

10. Sanderson AR, Leid JG, Hunsaker D. Bacterial biofilms on the sinus mucosa of human subjects with chronic rhinosinusitis. Laryngoscope. 2006;116:1121-1126.

11. Polzehl D, Moeller P, Riechelmann H, Perner S. Distinct features of chronic rhinosinusitis with and without nasal polyps. Allergy. 2006;61:1275-1279.

12. Arjomandi H, Gilde J, Zhu S, Delaney S, Hochstim C, Mazhar K, et al. Relationship of eosinophils and plasma cells to biofilm in chronic rhinosinusitis. Am J Rhinol Allergy. 2013;27:e85-e90.

13. Bjarnsholt $\mathrm{T}$. The role of bacterial biofilms in chronic infections. APMIS Suppl. 2013;136:1-51.

14. Psaltis AJ, Ha KR, Beule AG, Tan LW, Wormald PJ. Confocal scanning laser microscopy evidence of biofilms in patients with chronic rhinosinusitis. Laryngoscope. 2007;117:13021306.

15. Papadopoulou D, Dabrowska A, Harries PG, Webb JS, Allan RN, Salib RJ. Evaluation of a Bioengineered Honey and Its Synthetic Equivalent as Novel Staphylococcus aureus Biofilm-Targeted Topical Therapies in Chronic Rhinosinusitis. Am J Rhinol Allergy. 2020;34:80-86.

16. Lundberg JO. Nitric oxide and the paranasal sinuses. Anat Rec (Hoboken). 2008;291:1479-1484.

17. Suh JD, Ramakrishnan V, Palmer JN. Biofilms. Otolaryngol Clin North Am. 2010;43:521-530, viii.

18. Foreman A, Psaltis AJ, Tan LW, Wormald PJ. Characterization of bacterial and fungal biofilms in chronic rhinosinusitis. Allergy Rhinol (Providence). 2010;1:10.

19. Healy DY, Leid JG, Sanderson AR, Hunsaker DH. Biofilms with fungi in chronic rhinosinusitis. Otolaryngol Head Neck Surg. 2008;138:641-647.

20. Foreman A, Wormald PJ. Different biofilms, different disease? A clinical outcomes study. Laryngoscope. 2010;120:1701-1706.

21. Vestby LK, Grønseth T, Simm R, Nesse LL. Bacterial Biofilm and its Role in the Pathogenesis of Disease. Antibiotics (Basel). 2020;9:59.

22. Tamashiro E, Antunes MB, Palmer JN, Cohen NA, AnselmoLima WT. Implications of bacterial biofilms in chronic rhinosinusitis. Braz J Infect Dis. 2009;13:232-235.

23. Dong D, Thomas N, Ramezanpour M, Psaltis AJ, Huang $\mathrm{S}$, Zhao Y, et al. Inhibition of Staphylococcus aureus and Pseudomonas aeruginosa biofilms by quatsomes in low concentrations. Exp Biol Med (Maywood). 2020;245:34-41.

24. Bendouah Z, Barbeau J, Hamad WA, Desrosiers M. Biofilm formation by Staphylococcus aureus and Pseudomonas aeruginosa is associated with an unfavorable evolution after surgery for chronic sinusitis and nasal polyposis. Otolaryngol Head Neck Surg. 2006;134:991-996.

25. Hochstim CJ, Choi JY, Lowe D, Masood R, Rice DH. Biofilm detection with hematoxylin-eosin staining. Arch Otolaryngol Head Neck Surg. 2010;136:453-456.

26. Soler ZM, Sauer DA, Mace J, Smith TL. Relationship between clinical measures and histopathologic findings in chronic rhinosinusitis. Otolaryngol Head Neck Surg. 2009;141:454-461.

27. Ferguson BJ. Categorization of eosinophilic chronic rhinosinusitis. Curr Opin Otolaryngol Head Neck Surg. 2004; 12:237-242.

28. Grayson JW, Cavada M, Harvey RJ. Clinically relevant 
phenotypes in chronic rhinosinusitis. J Otolaryngol Head Neck Surg. 2019;48:2.

29. Rivero A, Liang J. Anti-IgE and Anti-IL5 Biologic Therapy in the Treatment of Nasal Polyposis: A Systematic Review and Meta-analysis. Ann Otol Rhinol Laryngol. 2017;126:739747.

30. van Drunen CM, Reinartz S, Wigman J, Fokkens WJ. Inflammation in chronic rhinosinusitis and nasal polyposis. Immunol Allergy Clin North Am. 2009;29:621-629.

31. Lee VS. Topical Irrigations for Chronic Rhinosinusitis. Immunol Allergy Clin North Am. 2020;40:317-328.

32. Farag AA, Deal AM, McKinney KA, Thorp BD, Senior BA, Ebert CS Jr, et al. Single-blind randomized controlled trial of surfactant vs hypertonic saline irrigation following endoscopic endonasal surgery. Int Forum Allergy Rhinol. 2013;3:276-280.

33. Rudmik L, Hoy M, Schlosser RJ, Harvey RJ, Welch KC, Lund $\mathrm{V}$, et al. Topical therapies in the management of chronic rhinosinusitis: an evidence-based review with recommendations. Int Forum Allergy Rhinol. 2013;3:281298.

34. Brown CL, Graham SM, Cable BB, Ozer EA, Taft PJ, Zabner J. Xylitol enhances bacterial killing in the rabbit maxillary sinus. Laryngoscope. 2004;114:2021-2024.

35. Kurola P, Tapiainen T, Sevander J, Kaijalainen T, Leinonen M, Uhari M, et al. Effect of xylitol and other carbon sources on Streptococcus pneumoniae biofilm formation and gene expression in vitro. APMIS. 2011;119:135-142.

36. Weissman JD, Fernandez F, Hwang PH. Xylitol nasal irrigation in the management of chronic rhinosinusitis: a pilot study. Laryngoscope. 2011;121:2468-2472.

37. Jervis-Bardy J, Foreman A, Bray S, Tan L, Wormald PJ. Methylglyoxal-infused honey mimics the antiStaphylococcus aureus biofilm activity of manuka honey: potential implication in chronic rhinosinusitis. Laryngoscope. 2011;121:1104-1107.

38. Kilty SJ, Duval M, Chan FT, Ferris W, Slinger R. Methylglyoxal: (active agent of manuka honey) in vitro activity against bacterial biofilms. Int Forum Allergy Rhinol. 2011;1:348-350.

39. Lu J, Turnbull L, Burke CM, Liu M, Carter DA, Schlothauer $\mathrm{RC}$, et al. Manuka-type honeys can eradicate biofilms produced by Staphylococcus aureus strains with different biofilm-forming abilities. PeerJ. 2014;2:e326.

40. Lusby PE, Coombes AL, Wilkinson JM. Bactericidal activity of different honeys against pathogenic bacteria. Arch Med Res. 2005;36:464-467.

41. Lim DJ, Skinner D, Mclemore J, Rivers N, Elder JB, Allen M, et al. In-vitro evaluation of a ciprofloxacin and azithromycin sinus stent for Pseudomonas aeruginosa biofilms. Int Forum Allergy Rhinol. 2020;10:121-127.

42. Cervin AU. The Potential for Topical Probiotic Treatment of Chronic Rhinosinusitis, a Personal Perspective. Front Cell Infect Microbiol. 2018;7:530. 